\title{
FORMACIONES BOSCOSAS EN SIERRA MORENA ORIENTAL (ANDALUCIA, ESPAÑA)
}

\author{
Eusebio CANO \& Francisco VALLE
}

RESUMEN: En el presente trabajo se estudia la fitosociología de los bosques localizados en Sierra Morena Oriental (comarca de Andújar, Jaén, Andalucía), comentando las siguientes asociaciones: Pyro bourgaeanae-Quercetum rotundifoliae Rivas Martínez 1987 (de la que se proponen como nuevas la subas. quercetosum fagineae Velasco ex Cano \& Valle y myrtetosum communis Cano \& Valle); Sanguisorbo agrimonioidis-Quercetum suberis Rivas Goday 1959 y Arbuto unedonis-Quercetum pyrenaicae Rivas Goday ex Rivas Martínez 1987, de la que se describe como nueva la subas. blechnetosum spicantis Cano \& Valle.

Palabras clave: Fitosociología, bosques.

ABSTRACT: In this paper we have studied the phytosociology of the forests wich are located in east Sierra Morena (Jaén, Andalucía), proposing the following syntaxa as new: Pyro bourgaeanae-Quercetum rotundifoliae Rivas Martínez 1987 subas. quercetosum fagineae Velasco ex Cano \& Valle and myrtetosum communis Cano \& Valle, and Arbuto unedonis-Quercetum pyrenaicae Rivas Goday ex Rivas Martínez 1987 subas. blechnetosum spicantis Cano \& Valle.

Key words: Phytosociology, forests.

\section{N T R O D U C C I O N}

La zona estudiada se localiza en el término municipal de Andújar (Jaén), dentro del subsector Marianense (sector Mariánico-Monchiquense, provincia Luso-Extremadurense), contactando con la provincia Castellano-Maestrazgo-Manchega (Rivas Martínez, 1982). La topografía es poco abrupta, siendo su altitud máxima de 1.290 metros (Burcio del Pino). Los sustratos dominantes son cuarcitas y pizarras y los suelos más extendidos cambisoles eútricos y dístricos, regosoles eútricos, litosoles y luvisoles (Aguilar \& al., 1987). El piso bioclimático dominante es el mesomediterráneo, con ombroclima seco-subhúmedo. Todo esto posibilita las siguientes series de vegetación (Rivas Martínez, 1987): Pyro bougaeanae-Querçeto rotundifoliae sigmetum, Sanguisorbo agrimonioidis-Querceto suberis sigmetum y Arbuto unedonisQuerceto pyrenaicae sigmetum. 
Pyro bourgaeanae-Quercetum rotundifoliae Rivas Martínez 1987 (Tabla 1; inv.1 a 13)

Se trata del típico encinar silicícola mesomediterráneo Luso-Extremadurense que se desarrolla sobre regosoles y cambisoles eútricos. En esta zona ocupa altiplanos donde la sequedad estival y los fríos invernales son más marcados. En orientaciones norte y barrancos frescos, donde las condiciones son más mesofíticas, la comunidad se enriquece en Quercus faginea, Pistacia terebinthus y Phillyrea latifolia, propias de la subas. quercetosum fagineae Velasco nova (tabla 1, inv. 7 al 10; síntipo inv. 7), que aunque comentada en numerosas ocasiones, no había sido tipificada (Cano, 1988).

En la porción meridional del territorio, ocupando laderas soleadas de los barrancos, son frecuentes especies como Myrtus communis, Olea europea var. sylvestris o Smilax aspera, de fuerte matiz termófilo y que actúan como diferenciales de la subas. myrtetosum communis Cano \& Valle nova (tabla 1, inv. 11 al 13; síntipo inv. 11); esta comunidad marca el tránsito hacia el Myrto-Quercetum rotundifoliae (Rivas Goday 1964) Rivas Martínez 1982 que se localiza en las proximidades del río Guadalquivir, en los alrededores de Andújar.

La degradación de estos bosques da lugar a un coscojal perteneciente al Hyacinthoido-Quercetum cocciferae (Rivas Goday 1959) Peinado \& Martínez Parras 1985 o a un retamal (Cytiso bourgaei-Retametum sphaerocarpae Rivas Martínez \& Belmonte inéd.) si la desforestación es muy acusada. En lugares ocupados por encinaresquejigares del Pyro-Quercetum rotundifoliae subas. quercetosum fagineae pueden presentarse como primera etapa de sustitución madroñales (Phillyreo-Arbutetum unedi Rivas Goday \& F. Galiano in Rivas Goday \& al. 1959). Sobre suelos más erosionados se localiza un jaral (Genisto hirsutae -Cistetum ladaniferi Rivas Goday 1955 em. Rivas Martínez 1979); éste corresponde a la subas. cistetosum monspeliensis Rivas Goday 1955 en las situaciones más térmicas y a la subas. cistetosum populifolii Pérez Chiscano inéd. en los lugares más húmedos. (Esquema 1).

Sanguisorbo agrimonioidis-Quercetum suberis Rivas Goday 1959 (Tabla 2; inv. 1 a 9)

Se localizan estos bosques en las umbrías, así como en laderas orientadas al sur por encima de los 700 metros, pero siempre bajo ombroclimas subhúmedos y resguardados de los fríos vientos meseteños. Ocupan suelos bien coservados, tipo luvisoles crómicos o cambisoles dístricos, con un horizonte superior con abundante humus forestal muy ácido.

A pesar de que se han descrito numerosas subasociaciones de este sintaxon, nosotros no hemos detectado ninguna debido a las características tan homogéneas donde se dan estas formaciones en nuestro territorio. Sí observamos la presencia de plantas térmicas en algunos inventarios, que marcan el tránsito a los alcornocales termófilos del Oleo-Quercetum suberis Rivas Goday \& al. 1973 ex Rivas Martínez \& al. 1980 .

Como primer estadío de degradación de estos ecosistemas se presenta el ma- 
droñal Phillyreo-Arbutetum unedi subas. arbutetosum o viburnetosum tini Pérez Chiscano 1976 (en lugares húmedos y frescos). Una posteior degradación de estas comunidades daría lugar respectivamente a jarales (Genisto-Cistetum ladaniferi subas. ericetosum australis Rivas Goday 1955) o a brezales-jarales (Polygalo microphyllaeCistetum populifolii Rivas Goday 1974). Por último en suelos ya muy degradados el brezal perteneciente a la as. Halimio ocymoidis-Ericetum umbellatae Rivas Goday 1964, representa el último estadío en la serie de vegetación. (Esquema 1).

Arbuto unedonis-Quercetum pyrenaicae Rivas Goday ex Rivas Martínez 1987. (Tabla 3; inv. 1 al 6)

Cuando las condiciones se hacen más mesófitas (lugares donde se condensan las nieblas, al lado de grandes canchales cuarcíticos y sobre suelos muy bien conservados, tipo luvisol crómico) los alcornocales son sustituidos por melojares con madroños. En los puntos más altos del territorio se observa el inicio de la serie supramediterránea Sorbo torminalis -Querceto pyrenaicae sigmetum sin que se presenten restos del melojar pero sí alguna de las comunidades propias de este sinecosistema (piornales del Adenocarpetum argyrophylli Rivas Martínez \& Belmonte inéd.).

Hemos reconocido, además de la subas. típica (quercetosum pyrenaicae), la subas. blechnetosum spicantis Cano \& Valle nova (tabla 3, inv. 5 y 6; síntipo inv. 5 ), para aquellos lugares con orientaciones norte, situados en barrancos umbríos donde las condiciones mesofíticas son muy marcadas; nuestra subasociación marca el tránsito entre el ecosistema climatófilo del melojar y el edafófilo de la aliseda (Scrophulario scorodoniae-Alnetum glutinosae Br.-Bl., Pinto da Silva \& Rozeira 1956 nom inv.

Las comunidades procedentes de la degradación de estas formaciones se corresponden con las facies más mesófitas de los alcornocales vistos con anterioridad, es decir, madroñales del Phillyreo-Arbutetum subas. viburnetosum tini y jarales-brezales del Polygalo-Cistetum populifolii. (Esquema 1).

\section{B I B L I O G R A F I A}

AGUilar, J., DElgado CALVO FlorES, G., DELGADO CALVO FlORES, R., DELGADO RODRIGUEZ, M., FERNANDEZ GARCIA, J., NOGALES, R., ORTEGA, E., PARRAGA, J., SAURA VILCHEZ, I., SIERRA, C. \& SIMON, M. -1987- Memoria del mapa de suelos de la provincia de Jaén a escala 1:200.000. Servicio Publicaciones Universidad de Granada.

CANO, E. -1988- Estudio fitosociológico de la Sierra de Quintana (Sierra Morena, Jaén). Tesis Doctoral. Universidad de Granada.

RIVAS MARTINEZ, S. -1982- Etages bioclimatiques, secteurs chorologiques et séries de végétation de l'Espagne méditerranéenne. Ecologia Mediterranea, 8:275-288.

RIVAS MARTINEZ, S. -1987- Memoria del mapa de las series de vegetación de España. I.C.O.N.A. Madrid.

(Aceptado para su publicación el 5 de febrero de 1990)

Dirección de los autores: Departamento de Biología Vegetal. Universidad de Granada. 


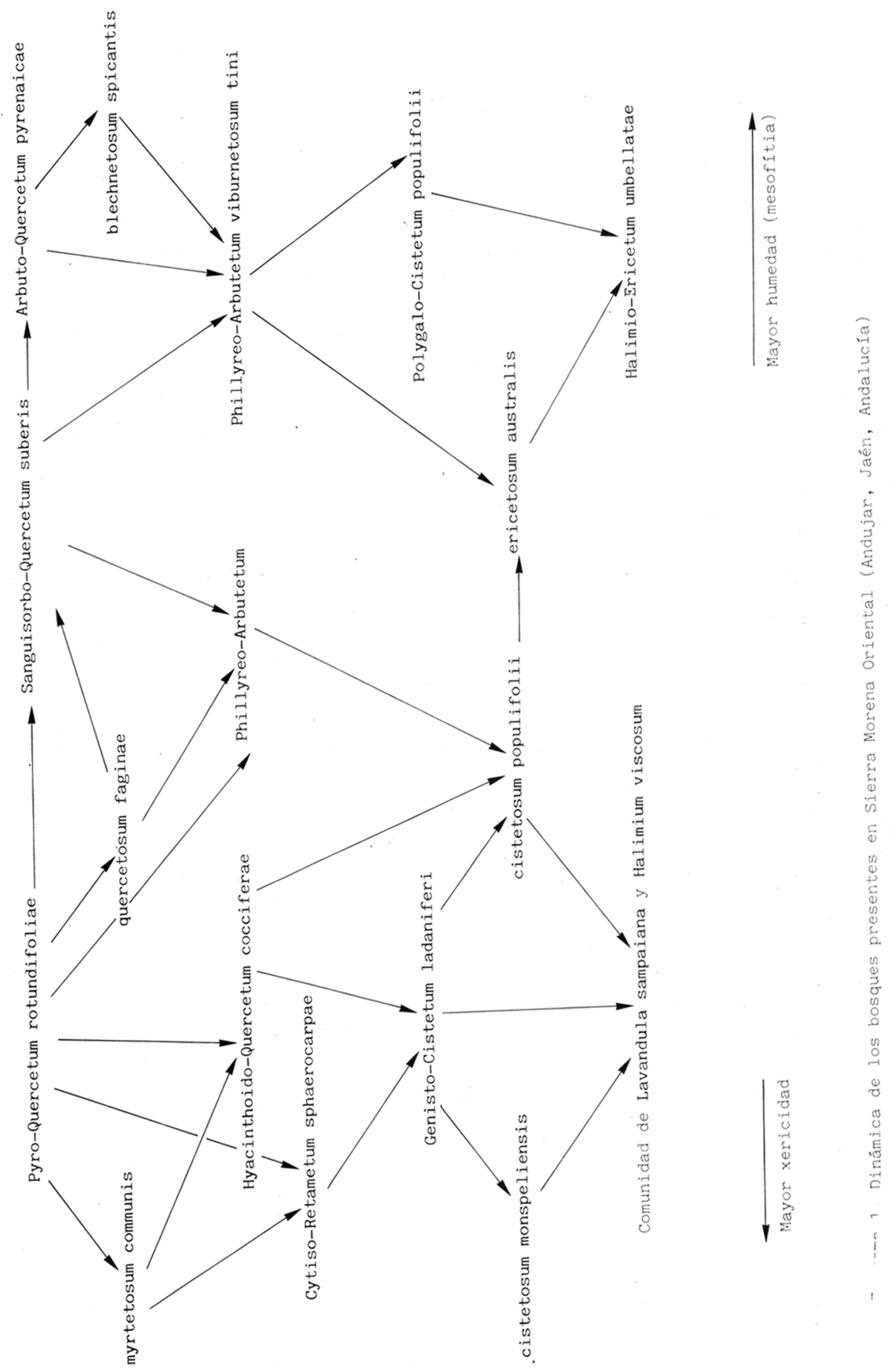


Pyro bourgaeanae-Q̄uercetun rotundifoliae Kiyas Martinez $198 \overline{7}$

quercetosun rotundifoliae, quercetosun faginea Velasco inéd., avrtetosun cosaunis nova

(Paeonio broteroi-üuercenion rotundifoliae, Üuercion rotundifoliae, Guercetalia ilicis, Guercetea ilicis/.

\author{
Altitud $1=100$. \\ Arez $\pi$ in \\ Inclinacion \% \\ Grientación \\ Nümero de especies \\ Nümero de orden \\ Caracteristicas de asociacion \\ v de alianza: \\ Guercus rotundifolia \\ Pyrus bourgaeana \\ Praeonia broteroi \\ Kuscus aculeatus \\ Doronicu plantagineus
}

\begin{tabular}{|c|c|c|c|c|c|c|c|c|c|c|c|}
\hline & $0 i$ & 60 & 73 & b8̈ & bil & bu & 60 & 68 & 56 & 38 & 55 \\
\hline$\phi$ & 300 & 460 & 360 & 400 & 400 & 400 & 100 & the & 150 & Sw0 & 150 \\
\hline & 25 & - & 5 & 20 & 10 & 15 & 20 & 20 & 25 & 10 & 30 \\
\hline & 5 & - & 5 & NE & $\mathrm{H}$ & 5 & W & HE & $\mathrm{HW}$ & H & \\
\hline & 10 & 9 & & 9 & o & 11 & 11 & 11 & 17 & 14 & \\
\hline & 2 & 3 & & 5 & 6 & $\bar{i}$ & 8 & $\overline{4}$ & 10 & 11 & 12 \\
\hline
\end{tabular}

$\begin{array}{lllllllllllll}3-3 & 3-3 & 3-3 & 3-3 & 4-4 & 4-4 & 3-4 & 3-5 & 3-3 & 3-3 & 3-3 & 3-3 & 3-3\end{array}$

$3-3 \quad 1-1 \quad 3-3 \quad 2-21-1.1-1 \quad 1-1 \quad 1-1+1-1.1-1$

Diferenciales de la subagociacion

quercetosu. fagineae:

Quercus faginea

Phillyrea latifolia

Fistacia terebinthus

jiferenciales de la subasociacion

ayrtetosun comunis:

Myrtus conounis

Olea syivestris

Sailax aspera

Laracteristicas de unidades

superiores:

Phillyrea angustifolia

Pistacia lentiscus

Daphne gnidiun

Ëuercus cocciferd

Arbutus unedo

Compañeras:

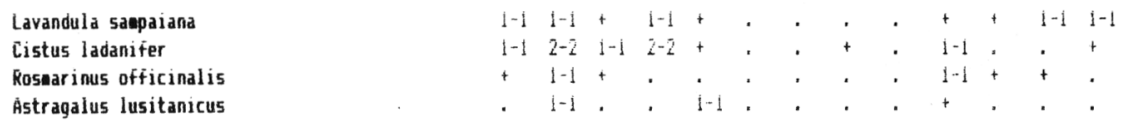

Hidemás: Urginea earitiaa + en 1; Thyous mastichina + y Cistus populifolius + en 5 ; Crataegus monogya subsp. brevispina + en b: Viburnus tinus 1-1. Erica arborea $1-1$ en 8 : Asparagus acutifolius + y Crataegus anogyna subsp. brevispina 1-1

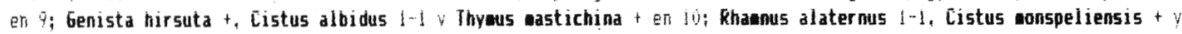
Cistus salvifolius + en II: Cistus onspeliensis $1-1$ en 13.

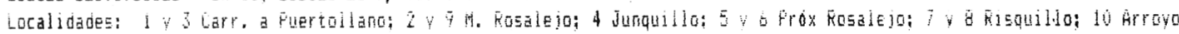
de los Peces: 11 y 12 Garganta de Valquemado; 13 Baranco del Fresnedoso (fuera ya del territor 10 . 
Sanguisorbo agrimonioidis-Duercetun suber is Riyas Godav 1959 (Üuercenion broteroi, Guercion broteroi, Guercetalia ilicis, Uuercetea ilicisi.

Altitud $1=10 \mathrm{~m}$.

Area 2

Incilinacion :

Orientacion

Numero de especies

Numero de orden

Caracteristicas de aso-

ciacion y alianza:

Quercus suber

Sanguisor ba hybrida

Ruscus aculeatus

Doronicu plantagineun

Paeonia broteroi

Caracteristicas de uni-

dades superiores:

Phillyrea angustifolia

Guercus faginea

Arbutus unedo

Daphne gnidiu:

Erica arborea

Guercus rotundifolia

Juniperus oxycedrus

hyrtus coneunis

Rubia peregrina

Fistacia lentiscus

Compañีย

Astragalus lusitanicus

Cistus ladanifer

Cistus populifolius

Cistus salvifolius

Tanus coneunis

Lavandula saepaiana

Fteridiua aquilinu

$\begin{array}{lllllllll}90 & 76 & 111 & 103 & 76 & 75 & 76 & 78 & 102 \\ 400 & 400 & 400 & 400 & 400 & 200 & 200 & 400 & 200 \\ 20 & 18 & 24 & 24 & 15 & 30 & 8 & 18 & 30 \\ 5 & 5 & 5 E & 51 & 5 & N 4 & 5 & 5 & 5 \\ 14 & 14 & 14 & 14 & 15 & 14 & 14 & 17 & 20 \\ 1 & 2 & 3 & 4 & 5 & 6 & 7 & 8 & 9\end{array}$

$$
\begin{aligned}
& 2-21-1++1-12-2+3+ \\
& \begin{array}{lllllllll}
1-1 & 3-3 & . & 1-1 & 1-1 & 3-3 & 3-3 & 1-1
\end{array} \\
& 2-2 \quad 2-2, \quad 2-32-21-1+1-1 \\
& 1-12-2+\text {. } 1-1,+++ \\
& 2-31-1+, 2-2,1-\overline{2},+ \\
& \text {. } 1-1+.+1-1+ \\
& +.1-1, \cdot+2-\bar{i}+ \\
& 1-1+.,+.+1-1 . \\
& \cdot+\cdot+1-1 .
\end{aligned}
$$

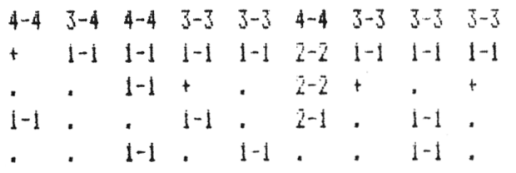

Además: Canpanula lusitanica + en 2; Urginea aritina + en 3; Äsphodelus albus y Arrhenatheru elatius subsp. bulbosun + en 4 ; Psoralea bituninosa $t$, Campanula lusitanica + $y$ Lotus parviflorus + en 5 ; Kubus ulaifolius + en $\bar{i}$; Duercus pyrenaica $\bar{z}-\bar{z}$ y Fistacia terebinthus + en $\bar{q}$.

Localidades: 1,2,3,4,5,7 y $\bar{y}$ Sierra üuintana; o M. Kosalejo: ă El Fanizal. 


\section{Tabla 3}

Arbuto unedonis-Guercetue pyrenaicae Rivas boday ex Rivas Martinez 1987 quercetosu pyrenaica, blechnetosun spicantis nova.

Güercenion pyrenaicae, Euercion robori-pyrenaicae, Buercetalia roboripetraeae, Ouerco-Fagenea, Buerco-Fagetea)

$\begin{array}{lllllll}\text { Altitud } 1=10 \text {.1.. } & 80 & 110 & 102 & 102 & 74 & 74 \\ \text { Area } 2 & 100 & 600 & 400 & 300 & 400 & 200 \\ \text { Inclinacion \% } & 25 & 15 & 20 & 30 & 15 & 15 \\ \text { Örientación } & 15 & 5 & 5 & 5 & N & N \\ \text { Número de especies } & 10 & 8 & 16 & 12 & 14 & 11 \\ \text { Numero de orden } & 1 & 2 & 3 & 4 & 5 & 6\end{array}$

Caracteristicas de asociacion

y unidades superiores:

Ouercus pyrenaica

Arbutus unedo

Ácer monspessulanu:

Tamus coneunis

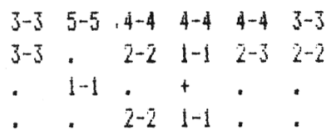

Diferenciales de la subaso-

ciacion blechnetosun spi-

cantis:

Blechnue spicant

Ossunda regalis

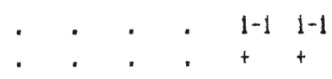

Compañeras:

Cistus ladanifer

Erica arborea

Daphne gnidiuu

Ouercus rotundifolia

Ouercus faginea

Phillyrea angustifolia

Juniperus oxycedrus

Pistacia terebinthus

Caepanula rapunculus

Clinopodiue vulgare

Vincetoxicue nigru.

Viburnue tinus

Ảstragalus Iusitanicus

Digitalis ariana

Paeonia broteroi

$$
\begin{aligned}
& +\quad 1-1++1-1 \\
& 1-1 \cdot 2-2 \cdot 2-21-1 \\
& \text { - }+2-z+1-1 \\
& +\quad 1-1+1-1 . \\
& 1-1.2 .2-23-3 \\
& 1-1 \cdot 1-1 \cdot 1-1 \text {. } \\
& 1-1,1-1+. . \\
& \text {. }+2-2 \cdot 1-1 \text {. } \\
& \text {. }+1-1 .+ \\
& \text {. }+1-1 \cdot 1-1 . \\
& \text {. } 2-2 \text {. }+1-1 \\
& 2-2, \quad 2-2 \cdot \text {. } \\
& \text {. . 1-1 1-1. . } \\
& \text { - } 2-2.1-1 .
\end{aligned}
$$

Además: Uuercus suber $1-1$ en 3 ; Pteridiun aquilinus + en 5 ; Teucriu fruticans + en 3; Lavandula luisieri + en 1; Cistus salvifolius + en 3 y Haliniua ocynoides + en 5 .

Localidades: 1, 3 y 4 Sierra de Uuintaria: 2 Eurcio del Fino lšierra de Uuintana); 5 y 6 Sierra de Madrona (Finca de Aulagas). 